VOL. $38(1988) \quad[321-323]$

\title{
POINT AT INFINITY
}

\author{
NiEL SHell
}

\begin{abstract}
We adjoin a point at infinity to a field with a ring topology and extend the topology by taking complements of bounded sets as neighbourhoods of infinity. We note some elementary relations between the topology on the field and the topology on the field with infinity adjoined.
\end{abstract}

Let $\tau$ be a Hausdorff ring topology on a (commutative) field $K$, and let $K_{\infty}=$ $K \cup\{\infty\}$, where $\infty$ is any element not in $K$. We let

$$
\tau(\infty)=\tau \cup\left\{K_{\infty} \backslash B: B \text { is } \tau \text {-closed and bounded }\right\}
$$

We denote by $A^{*}$ the nonzero elements of a subset $A$ of $K_{\infty}$. We extend the algebraic operations by defining $0^{-1}=\infty, \infty^{-1}=0$, and $a \cdot \infty=\infty \cdot a=\infty$ for $a \in\left(K_{\infty}\right)^{*}$.

Certainly, $\tau(\infty)$ is a topology inducing $\tau$ on $K$ (see [4]), and a subset of $K_{\infty}$ is a neighbourhood of $\infty$ if and only if its complement is a $\tau$-bounded subset of $K$. $\left(K_{\infty}, \tau(\infty)\right)$ is Hausdorff if and only if $(K, \tau)$ is locally bounded; and $\left(K_{\infty}, \tau(\infty)\right)$ is a compact Hausdorff if and only if $(K, \tau)$ is a locally compact Hausdorff space. For locally compact fields $\left(K_{\infty}, \tau(\infty)\right)$ is the one-point compactification of $K$.

Let $\mathcal{N}$ and $\mathcal{B}$ denote the neighbourhood filter at zero in $K_{\infty}$ and the collection of bounded subsets of $K$, respectively. We define the maps $\mathbf{I}$ and $\mathbf{C}$ from the power set of $K_{\infty}$ to itself:

$$
\text { I: } E \mapsto E^{-1} \quad \mathrm{C}: E \mapsto K_{\infty} \backslash E .
$$

Then $\mathbf{I}$ and $\mathbf{C}$ are self-inverse bijections that commute with each other. Also $\mathbf{C N}$ and $\mathrm{CB}$ are the collection of sets bounded away from zero and the neighbourhood filter at infinity, respectively.

LEMMA. In a field with a Hausdorff ring topology, the inverse of a bounded set is bounded away from zero [3, p. 1129].

That is, in the adjunction of infinity to any field with a Hausdorff ring topology, inversion is continuous at zero.

ProOF: The conclusions of the first and second statements of the lemma are equivalent, because both are equivalent to the containment $\operatorname{ICB} \subset \mathcal{N}$.

Received 10 December 1987

Copyright Clearance Centre, Inc. Serial-fee code: 0004-9729/88 \$A2.00+0.00. 
Considering the lemma, one might think that inversion is continuous at infinity in any field topology. However, this is not true, as the theorem below indicates. Recall that a field is said to be of type $V$ (locally retrobounded) if the inverse of a set bounded away from zero is bounded, and that type $V$ fields are important because type $V$ fields are exactly those whose topologies are induced by valuations ([2], see also [5]). Kaplansky [1] (see also [6, pp. 80-81]) observed that conditions (3) and (4) in the statement of the theorem below are each equivalent to being type $V$.

THEOREM. Let $\tau$ be a Hausdorff ring topology on a field $K$. The following statements are equivalent:

(1) $\tau$ is of type $V$;

(2) inversion is continuous at infinity;

(3) if $A$ and $B$ are subsets of $K$ which are bounded away from zero, then $A B$ is bounded away from zero;

(4) for each neighbourhood $U$ of zero there exists a neighbourhood $V$ of zero such that $x, y \in K \backslash U$ implies $x y \in K \backslash V$;

(5) $(K, \tau)$ is locally bounded and, for each bounded neighbourhood $V$ of zero, there exists a bounded neighbourhood $U$ of zero such that $x, y \in$ $K \backslash U$ implies $x y \in K \backslash V$;

(6) $(K, \tau)$ is locally bounded and multiplication on $\left[\left(K_{\infty}\right)^{*}\right]^{2}$ is continuous at $(\infty, \infty)$.

Proof: (1) and (2) are equivalent: Statement (1) is ICN $\subseteq \mathcal{B}$, and statement (2) is $\mathbf{I}^{-1} \mathcal{N} \subseteq \mathbf{C B}$.

It was already noted that statements (1), (3) and (4) are equivalent.

(5) is equivalent to (6): For locally bounded topologies the complements in $K_{\infty}$ of bounded neighbourhoods of zero form a neighbourhood base at infinity. The second condition in $(5)$, namely, that, given a bounded neighbourhood $V$ of zero, there exists a bounded neighbourhood $U$ of zero such that

$$
(\mathbf{C} U)(\mathbf{C} U) \subseteq(\mathbf{C} V)
$$

is exactly the statement of continuity phrased in terms of the base at infinity described above.

(6) implies (4): Suppose (6) holds and a neighbourhood $U$ of zero in $K$ is given. Let $W$ be any bounded neighbourhood of zero in $K$. The continuity of multiplication implies the existence of a bounded subset $B$ of $K$ such that $(\mathbf{C} B)(\mathbf{C} B) \subseteq \mathbf{C W}$. If 
$x \in K^{*}$ and $x B \subseteq U$, then we may satisfy condition (4) by letting $V=x^{2} W$ :

$$
\begin{gathered}
\left(\mathbf{C} x^{-1} U\right)\left(\mathbf{C} x^{-1} U\right) \subseteq(\mathbf{C} B)(\mathbf{C} B) \subseteq \mathbf{C W} \\
\left(x \mathbf{C} \boldsymbol{x}^{-1} U\right)\left(x \mathbf{C} x^{-1} U\right) \subseteq \mathbf{C}\left(x^{2} W\right) \\
(\mathbf{C} U)(\mathbf{C} U) \subseteq \mathbf{C}\left(x^{2} W\right)
\end{gathered}
$$

(1) and (4) imply (5): From (1) we see ([2, Theorem 8]) that $(K, \tau)$ is locally bounded. Let $U$ be any bounded neighbourhood of zero. Choose a neighbourhood $W$ of zero such that $(\mathbf{C} U)(\mathbf{C} U) \subset \mathbf{C W}$. Suppose a bounded neighbourhood $V$ of zero is given. Choose neighbourhoods $W_{1}$ and $W_{2}$ of zero such that $W_{1} V \subseteq W$ and $W_{2} W_{2} \subseteq W_{1}$. Then $x^{2} V \subseteq W$ for each $x \in W_{2}^{*}$. For such $x$,

$$
\left(\mathbf{C} x^{-1} U\right)\left(\mathbf{C} x^{-1} U\right) \subseteq \mathbf{C} x^{-2} W \subseteq \mathbf{C} V
$$

In (5) the requirement that $V$ be bounded cannot be omitted, since there clearly is no solution $U$ if $V=K$ and $\tau$ is not discrete. Nor can we omit the requirement that $U$ be bounded, since clearly $U=K$ is a solution for any neighbourhood $V$ in any ring topology on $K$. Finally, the requirement that the field be locally bounded cannot be onitted from (5), since this modification of statement (5) would then vacuously be true in any field that is not locally bounded.

Intuitively, (2) in the theorem indicates that a field is type $V$ if and only if the inverse of a big element is small, and (6) indicates that a locally bounded field is type $V$ if and only if a product of large elements is large.

\section{REFERENCES}

[1] I. Kaplansky, 'Polynomials in topological fields', Bull. Amer. Math. Soc. 64 (1948), 909-916.

[2] H.J. Kowalsky and H. Dürbaum, 'Arithmetische Kennzeichnung von Korpertopologien', J. Reine Angew. Math. 191 (1953), 135-152.

[3] L. Nachbin, 'On strictly minimal topological division rings', Bull. Amer. Math. Soc. 55 (1949), 1128-1136.

[4] N. Shell, 'Near valuations' (to appear).

[5] N. Shilkret (Shell), 'One point adjunctions', Math. Student 42 (1974), p. 99.

[6] W. Więslaw, Topological Fields (Acta Univ. Waratislav. Mat. Fiz. Astronom. No. 43, 1982).

The City College of New York (CUNY)

Convent Avenue at 138 th Street

New York, NY 10031

United States of America. 